\title{
Near-infrared photometry of Galactic planetary nebulae with the VVV Survey ${ }^{\star}$
}

\author{
W. A. Weidmann ${ }^{1, \star \star}$, R. Gamen ${ }^{2, \star \star \star}$, P. A. M. van Hoof $^{3}$, A. Zijlstra ${ }^{4}$, D. Minniti ${ }^{5,6,7}$, and M. G. Volpe ${ }^{8}$ \\ ${ }^{1}$ Observatorio Astronómico Córdoba, Universidad Nacional de Córdoba, X5000BGP Cordoba, Argentina \\ e-mail: walter@mail.oac.uncor.edu \\ 2 Instituto de Astrofísica de La Plata, CCT La Plata-CONICET, Universidad Nacional de La Plata, Paseo del Bosque s/n 1900, \\ Argentina \\ e-mail: rgamen@fcaglp.unlp.edu.ar \\ 3 Royal Observatory of Belgium, Ringlaan 3, 1180 Brussels, Belgium \\ 4 Jodrell Bank Centre for Astrophysics, University of Manchester, Manchester M13 9PL, UK \\ 5 Departamento de Astronomía y Astrofísica, Pontificia Universidad Católica de Chile, Av. Vicuña Mackenna 4860, Casilla 306, \\ Santiago 22, Chile \\ e-mail: dante@astro.puc.cl \\ 6 Vatican Observatory, V00120 Vatican City State, Italy \\ 7 Departamento de Ciencia Fisicas, Universidad Andres Bello, Republica 4402 do Piso Santiago, Chile \\ 8 Instituto de Astronomía Teórica y Experimental, X5000BGP Cordoba, Argentina
}

Received 3 October 2012 / Accepted 20 February 2013

\section{ABSTRACT}

\begin{abstract}
Context. Planetary nebulae (PNe) are powerful tracers of evolved stellar populations. Among the 3000 known PNe in the Galaxy, about 600 are located within the 520 square-degree area covered by the VVV survey. The VVV photometric catalogue provides an important new dataset for the study of PNe, with high-resolution imaging in five near-infrared bands.

Aims. There are various colour-colour diagrams that can be obtained from the VVV filters. We investigate the location of PNe in these diagrams and the separation from other types of objects. This includes the new $Y-J$ vs. $Z-Y$ diagram.

Methods. Aperture photometry of known PNe in the VVV area was retrieved from source catalogues. Care was taken to minimise any confusion with field stars. The colours of the PNe we are determined for $\left(H-K_{\mathrm{s}}\right),(J-H),(Z-Y)$, and $(Y-J)$, and compared to stars and to other types of emission line objects. Cloudy photo-ionisation models were used to predict colours for typical PNe.

Results. We present near-infrared photometry for 353 known PNe. The best separation from other objects is obtained in the $\left(H-K_{\mathrm{s}}\right)$ vs. $(J-H)$ diagram. We calculated the emission-line contribution to the in-band flux based on a model for NGC 6720: we find that this is highest in the $Z$ and $Y$ bands at over 50\%, lower in the $J$ band at 40\%, and lowest in the $H$ and $K_{\mathrm{s}}$ bands at 20\%. A new view of PNe in the wavelength domain of the $Z$ and $Y$ bands is shown. Photo-ionisation models are used to explore the observed colours in these bands. The $Y$ band is shown to be dominated by He I $1.083 \mu \mathrm{m}$ and He II $1.012 \mu \mathrm{m}$, and colours involving this band are very sensitive to the temperature of the ionizing star.

Conclusions. The VVV survey represents a unique dataset for studying crowded and obscured regions in the Galactic plane. The diagnostic diagrams presented here allow one to study the properties of known PNe and to uncover objects not previously classified.
\end{abstract}

Key words. planetary nebulae: general - infrared: ISM - catalogs

\section{Introduction}

Planetary nebulae $(\mathrm{PNe})$ are luminous and short-lived products of evolved low-mass stars $\left(0.8 M_{\odot}<M<8 M_{\odot}\right)$. The stars eject much of their envelopes during a phase of strong mass loss at the end of the asymptotic giant branch (AGB). The ejecta become ionised after a brief transition phase, when the star rapidly increases in temperature, the ionised ejecta form a visible planetary nebula. The ejected material is enriched in a variety of elements ( $\mathrm{He}, \mathrm{C}, \mathrm{N}, \mathrm{O}, \mathrm{Ne}, \mathrm{Mg}$, s-process elements), through nuclear burning and dredge-up during the AGB phase. The transfer of this gas to the interstellar medium affects the overall chemical evolution of the Galaxy. PNe also provide information about

* Tables 3-5 are only available in electronic form at the CDS via anonymous ftp to cdsarc.u-strasbg. fr (130.79.128.5) or via http://cdsarc.u-strasbg. fr/viz-bin/qcat?]/A+A/552/A74

$\star \star$ Member of Carrera del Investigador CONICET, Argentina.

$\star \star \star$ Member of Carrera del Investigador CONICET, Argentina. the recent star-forming history in our Galaxy (e.g. Maciel \& Costa 2003). Thus, PNe are key objects in the study of stellar and galactic evolution.

The distribution of known Galactic PNe shows a lack of objects within the Galactic plane and towards the central regions of the Galaxy (e.g. Miszalski et al. 2008, Fig. 6c). This is caused by high extinction and by severe crowding. To obtain a better understanding of the whole Galactic PN population, deeper surveys are needed in these regions. Near-infrared (NIR) observations provide an important tool, because they are less affected by extinction and benefit from better seeing.

The NIR fluxes of PNe depend on a broad range of emission mechanisms, including warm dust continuum emission, ionic and atomic permitted and forbidden line transitions, and the underlying free-free and bound-free components of gaseous continuum emission. Continuum emission from cool stellar companions, or the PN central star itself, may also contribute. Broad band IR photometry of galactic PNe has been 
reported in the literature by several authors (e.g. Allen 1973; Whitelock 1985; Pena \& Torres-Peimbert 1987; Garcia-Lario et al. 1997). The complete and homogeneous 2 MASS survey has substantially increased the number of PNe with NIR observations (Ramos-Larios \& Phillips 2005; Phillips \& Zepeda-García 2009), but was limited by its 4 -arcsec spatial resolution. The VISTA Variables in the Via Lactea $\left(\mathrm{VVV}^{1}\right)$ public survey now provides deeper and higher resolution observational data, and will greatly improve the number of $\mathrm{PNe}$ observations in extincted and/or crowded regions of the sky (Minniti et al. 2010).

The past studies of PNe have used the $J H K_{\mathrm{s}}$ bands. The VVV survey adds two original bands, the $Z$ and $Y$ bands ${ }^{2}$. Therefore, our motivations are to improve the infrared photometry of PNe in order to contribute to the knowledge of stellar evolution, and to generate tools for characterising the NIR flux of PNe.

\section{The VVV observational database}

The VISTA 4.1m telescope at the Paranal Observatory (Emerson \& Sutherland 2010) is equiped with the instrument VIRCAM (VISTA InfraRed CAMera; Emerson et al. 2006; Dalton et al. 2006). The camera contains 16 near infrared detectors, of $2048 \times$ 2048 pixels each. The pixel size corresponds to 0.34 arcsec on the sky. There are gaps between the detectors so that a single exposure gives a non-contiguous sky coverage. Such a single exposure is called a pawprint. By combining 6 pawprints with appropriate offsets, a contiguous coverage of a field is achieved, where all pixels (apart from those at the edges) have at least two independent exposures. In the VISTA terminology such a field is called a tile; it covers a 1.64 square deg field of view, 1.5 square deg of which is covered by at least 2 pawprints.

The data reduction was carried out in the typical manner for infrared imaging. Details of the procedure are described in Irwin et al. (2004). The median image quality measured on the reduced VVV tile images is around $0.8^{\prime \prime}$ for $K_{\mathrm{s}}, 0.9^{\prime \prime}$ for the $J$-band, and up to $1.0^{\prime \prime}$ for the $Z$-band.

The data calibration is performed by the VISTA Data Flow System (VDFS) pipeline at the Cambridge Astronomy Survey Unit (CASU). The photometry is tied to unsaturated 2MASS stars present in the VVV images, even for the $Z$ and $Y$ filters (not observed by $2 \mathrm{MASS}$ ), for which colour equations are applied ${ }^{3}$. For more detail about photometric calibration see Gonzalez et al. (2011) for all 5 filters.

In this paper we use the magnitudes from the first data release of the VVV Survey (Saito et al. 2012). The VISTA Science Archive ${ }^{4}$ provides two different catalogues, vvvDetection and $v v v$ Source. The first one contains the individual detections for sources originating from multiframe images taken from the VVV. The second one lists the merged sources (in the five bands) from detections in $v v v$ Detection catalogue (Saito et al. 2012). In our catalogue, we used the Source-table, with a $2.8^{\prime \prime}$ aperture diameter.

The catalogue contains a flag to indicate the most probable morphological classification, in particular " -1 " is used to denote stellar objects, " -2 " borderline stellar, " 0 " is noise, and " 1 " is

\footnotetext{
1 http://vvvsurvey.org/

2 We found only a single paper using the $Y$ broad band (Miszalski et al. 2011).

3 More details about VISTA data processing is available from http://casu.ast.cam.ac.uk/surveys-projects/vista/ data-processing

4 http://horus.roe.ac.uk/vsa
}

used for non-stellar objects. There are also further flags: "-7", denoting sources containing bad pixels, and the flag " -9 " is used for saturated stars (Irwin et al. 2004). This makes it possible to reject some bad measurements and to identify extended emission sources.

Objects with $J<11.8, H<11.0$, or $K_{\mathrm{s}}<11.0$ are saturated. We considered $Z Y<12.5$ to indicate saturation, taking into account that the magnitudes in these two bands are probably not as well calibrated as those at $J H K_{\mathrm{s}}$ due to the extrapolation needed. The magnitudes at which saturation occurs are dependent on the fields, partly because of seeing, and partly because there are some differences in exposure times between the bulge $\left(-10^{\circ}<l<10^{\circ}\right.$ and $\left.-10^{\circ}<b<5^{\circ}\right)$ and disc $\left(-65^{\circ}<l<-10^{\circ}\right.$ and $\left.-2^{\circ}<b<2^{\circ}\right)$. We prefer to adopt the limits shown above.

The magnitudes from the aperture photometry are extracted from the catalogues following the equation mag $=\mathrm{zp}-($ airm -1$) \times C-2.5 \times \log _{10}(F / T)-\mathrm{AC}$, where $\mathrm{zp}=$ photometric zero-point for default extinction, airm $=$ air mass, $\mathrm{C}=$ default extinction in the passband, $\mathrm{F}=$ aperture flux, $\mathrm{T}=$ exposure times and $\mathrm{AC}=$ aperture correction in magnitudes.

We refer the reader to Saito et al. (2012) for a description of the photometric errors in the $Z Y J H K_{\mathrm{s}}$ filters as a function of the magnitude at different levels of crowding along the VVV area ${ }^{5}$.

\section{Sources of $0.8-2.1 \mu \mathrm{m}$ emission in planetary nebulae in the VVV filters}

For a better understanding of the results shown in this paper it is worth studying the characteristics of the near infrared emission observed in PNe. Whitelock (1985) gave a complete description of the principal emission sources in the $J, H, K_{\mathrm{s}}$ bands, thermal plasma continuum, thermal dust emission, hot stellar continuum, emission lines and cool stellar continuum from a companion. Their discussion is based on the SAAO filters which slightly differ from other systems. In particular, the SAAO $J$-band includes the strong HeI 1.083 micron line at 50\% transmission, which can dominate the flux in that band. The VISTA (and 2MASS) $J$-band filter excludes this line and it marginally intrudes instead in the $Y$-filter.

In the new photometric bands $Z$ and $Y$, the dust contribution is neglible. Emission from free-free (FF) and bound-free (BF) transitions is marginally weaker. The stellar continuum gains importance, however the major flux contribution comes from emission lines. The $Z$ band is dominated by [S III] at $0.9069 \mu \mathrm{m}$, and the $Y$ band by $\mathrm{Pa} \delta$ and $\mathrm{He}$ II at $1.0124 \mu \mathrm{m}$. Figure 1 shows the $Z Y J$ transmission curves, in comparison to the spectrum of IC 5117, a medium excitation-class planetary nebula.

The 2MASS $J$ band is somewhat wider than the VISTA $J$ band. But the dominant $\operatorname{Pa} \beta$ is covered by both filters, whilst neither filter contains other significant emission lines. Therefore, we do not expect a major discrepancy between both magnitudes.

IPHAS (the INT/WFC Photometric H-alpha Survey, Drew et al. 2005), its southern extension VPHAS, and DENIS (Deep Near-Infrared Southern Sky Survey, Epchtein et al. 1997) contain two optical bands that overlap in some spectral range with the $Z$ VISTA band: these are $i^{\prime}$ (centred at $0.7743 \mu \mathrm{m}$ ) and $I$ $(0.8000 \mu \mathrm{m})$ respectively. However, magnitudes obtained from these optical bands cannot be compared with the $Z$ one because the filters are too different.

To further test the emission mechanisms contributing to the VVV bands, we made use of the published photo-ionisation

\footnotetext{
5 Errors in the fluxes are based on Poissonian statistics. A comprehensive description is available at Cross et al. (2012).
} 


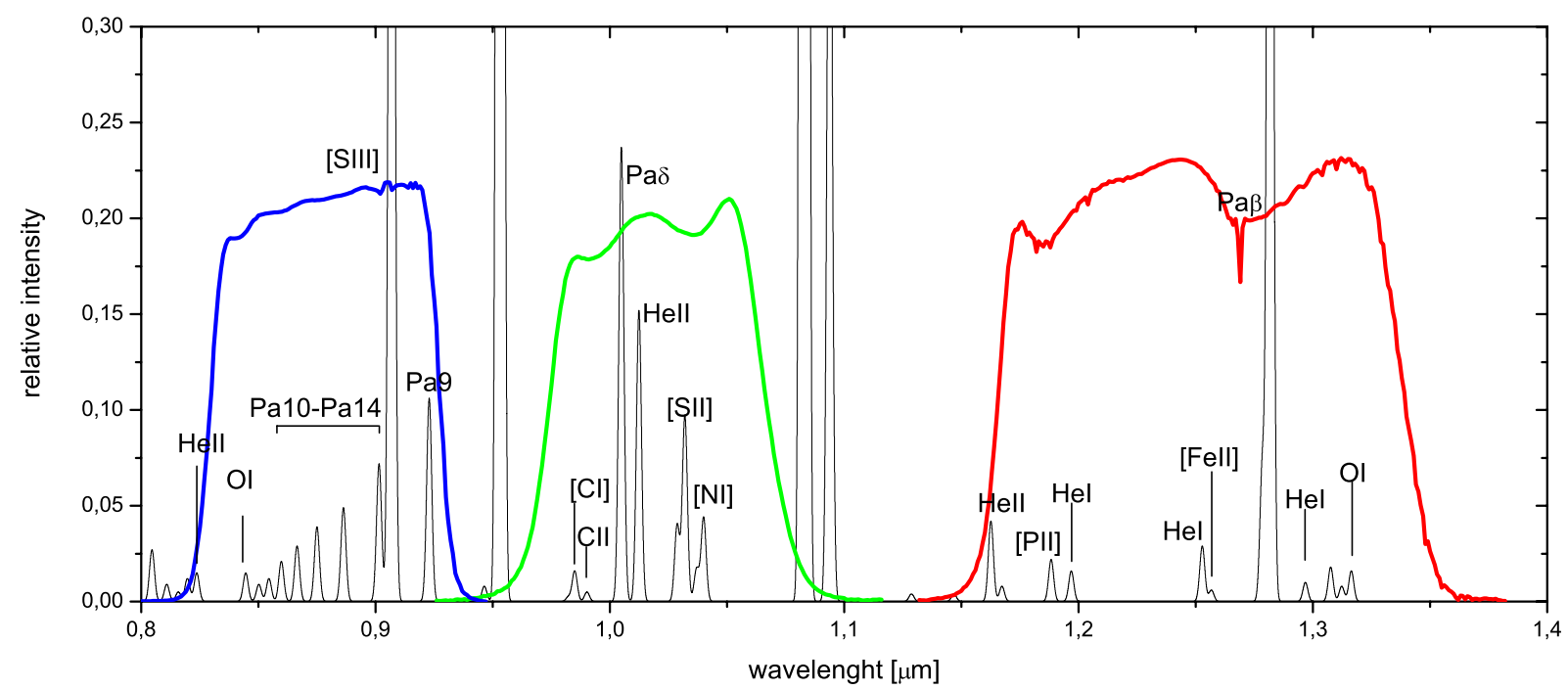

Fig. 1. Transmission curves for the $Z, Y$ and $J$ broad-band filters (the effective wavelengths are $0.878,1.021$ and $1.254 \mu$ m respectively), the filters are multiplied by a model atmosphere. The spectrum of the PN IC 5117 is superimposed (Rudy et al. 2001), scaled to $\mathrm{Pa} \beta=1$.

model of van Hoof et al. (2010) for NGC 6720, the Ring nebula. This is the archetypal planetary nebula; the nebula is bipolar, seen almost pole-on, optically thick to ionizing radiation except towards the poles. The Ring nebula has a central star which has already entered the cooling track, and in response to the fading of the star parts of the outer nebula have begun to recombine. The photo-ionisation model uses as ionizing source a H-Ni model stellar atmosphere from Rauch (2003) with a temperature of $T_{\text {eff }}=135 \mathrm{kK}$. The electron density of the model is $n_{\mathrm{e}}=416 \mathrm{~cm}^{-3}$. For comparison, IC 5117 shown in Fig. 1 has $T_{\text {eff }}=120 \mathrm{kK}$ and density $n_{\mathrm{e}}=9 \times 10^{4} \mathrm{~cm}^{-3}$ (Hyung et al. 2001). The model was calculated with version C10.00 of the Cloudy photo-ionisation code, last described by Ferland et al. (1998). The model incorporates the neutral region, to capture molecular hydrogen emission. We used this Cloudy model to calculate the free-free and bound-free continuum from both hydrogen and helium, and lines from ionised and neutral species, and from molecular hydrogen. The stellar continuum is also included but is not important at these long wavelengths and high stellar temperatures. The output model spectrum was convolved with the transmission curves from the VISTA filters combined with the telescope and instrument throughput and the detector sensitivity. Note that the model is for a nebula without interstellar reddening but does include a small amount of internal reddening.

Table 1 shows the detailed list of major lines which contribute to the in-band flux. The $Z$ and $J$-bands are dominated by one line. The $Y$ band also has one dominant line (He I) but it is located at the edge of the transmission curve. $H$ and $K_{\mathrm{s}}$ have a scattering of fainter lines. The total contribution of emission lines to the in-band flux is much higher for $Y$ and $Z$ than for the other three bands. The $Z$ and $Y$-filters have a strong line close to the edge of the transmission curve, and small changes in the filter responses can have significant effects on the line contributions. The specific line contributions will also depend on the excitation of the nebula especially for the $Z$-band.

\section{Results}

\subsection{Description of the sample}

There are 579 known PNe listed by Acker et al. (1992), Parker et al. (2006, MASH I), and Miszalski et al. (2008, MASH II) whose coordinates (Kerber et al. 2003) fall within the VVV area $^{6}$.

We retrieved the processed and calibrated $Z Y J H K_{\mathrm{s}}$ images (tiles) from the Cambridge Astronomical Survey Unit (CASU VIRCAM pipeline v1.17 ; Irwin et al. 2004) and also retrieved the MASH H $\alpha$ images (Parker et al. 2005). The planetary nebulae were identified from the $\mathrm{H} \alpha$ images using Kerber et al. (2003), the MASH catalogue and identification charts (Kinman et al. 1988; Beaulieu et al. 1999; Jacoby \& Van de Steene 2004). We identified the related VVV source taking into account its symmetry with respect to the $\mathrm{H} \alpha$ emission. The visual examination was essential. Cross-correlation between the VVV and $\mathrm{PNe}$ catalogues (Kerber et al. 2003 for example) did not give satisfactory results for many sources. Therefore, the VVV sources were associated with the PNe by eye. Up to $82 \%$ of the sample are closer than 1.4 arcsec to the catalogue coordinates of Kerber et al. (2003).

From the initial sample of $579 \mathrm{PNe}, 75$ are highly extended sources and these will be considered in future papers, and $123 \mathrm{PNe}$ could not be related to any source in the VVV images, perhaps because they have low surface brightness or the central star is too weak in the NIR bands. The remaining PNe analysed in this paper are related to sources in the VVV catalogues with NIR emission mainly enclosed in an area of 1.4 arcsec of radius (even in PNe of high surface brightness and appreciable angular size); in this sense we are observing the emission from the central regions of the nebula. The integrated flux may be underestimated where the nebula is larger than the aperture used here. See Appendix A for a discussion about the used aperture.

In our catalogue we divide the data into five categories (see Tables 3 and 5):

- Objects with photometric data (not necessarily in the five bands).

- NIR emission of the PN is detected in tile frames, but there are no photometric data in the VSA database (S/D).

- Extended objects, where the NIR emission is larger than $3^{\prime \prime}$ in diameter (ext).

6 The IPHAS survey (Viironen et al. 2009a) does not overlap with the VVV area.

7 Data release 1.1,

http://casu.ast. cam.ac.uk/surveys-projects/vista 
Table 1. Line contribution from the (unreddened) NGC 6720 model results.

\begin{tabular}{|c|c|c|c|c|}
\hline Line & $\begin{array}{c}\lambda \\
{[\mu \mathrm{m}]}\end{array}$ & $\begin{array}{c}\text { flux } \\
{\left[\log \mathrm{erg} \mathrm{cm}^{-2} \mathrm{~s}^{-1}\right]}\end{array}$ & $I($ line $) / I(\mathrm{H} \beta)$ & Transm. \\
\hline \multicolumn{5}{|c|}{$Z$ band $(0.878 \pm 0.097 \mu \mathrm{m})$} \\
\hline \multicolumn{5}{|c|}{ Line contribution $55 \%$} \\
\hline [S III] & 0.9069 & -10.762 & 0.1591 & 0.717 \\
\hline H I & 0.9229 & -11.576 & 0.0244 & 0.644 \\
\hline H 1 & 0.9015 & -11.716 & 0.0177 & 0.712 \\
\hline H 1 & 0.8863 & -11.842 & 0.0132 & 0.707 \\
\hline H 1 & 0.8750 & -11.956 & 0.0102 & 0.698 \\
\hline$[\mathrm{Cl} \mathrm{II}]$ & 0.8579 & -12.012 & 0.0089 & 0.677 \\
\hline \multicolumn{5}{|c|}{$Y$ band $(1.021 \pm 0.093 \mu \mathrm{m})$} \\
\hline \multicolumn{5}{|c|}{ Line contribution $55 \%$} \\
\hline He I & 1.083 & -10.342 & 0.4182 & 0.048 \\
\hline$[\mathrm{C} \mathrm{I}]$ & 0.985 & -11.166 & 0.0627 & 0.597 \\
\hline H I & 1.005 & -11.236 & 0.0534 & 0.645 \\
\hline He II & 1.012 & -11.305 & 0.0456 & 0.669 \\
\hline [S II $]$ & 1.033 & -11.382 & 0.0382 & 0.640 \\
\hline$[\mathrm{N} \mathrm{I}]$ & 1.040 & -12.026 & 0.0087 & 0.645 \\
\hline \multicolumn{5}{|c|}{$J$ band $(1.254 \pm 0.172 \mu \mathrm{m})$} \\
\hline \multicolumn{5}{|c|}{ Line contribution $39 \%$} \\
\hline H I & 1.282 & -10.772 & 0.1555 & 0.680 \\
\hline He II & 1.163 & -12.026 & 0.0087 & 0.226 \\
\hline Fe II & 1.257 & -12.109 & 0.0072 & 0.730 \\
\hline He I & 1.278 & -12.135 & 0.0067 & 0.668 \\
\hline \multicolumn{5}{|c|}{$H$ band $(1.646 \pm 0.291 \mu \mathrm{m})$} \\
\hline \multicolumn{5}{|c|}{ Line contribution $21 \%$} \\
\hline H I & 1.817 & -11.890 & 0.0118 & 0.040 \\
\hline H I & 1.736 & -12.031 & 0.0086 & 0.843 \\
\hline H I & 1.681 & -12.158 & 0.0064 & 0.850 \\
\hline $\mathrm{Fe}$ II & 1.644 & -12.182 & 0.0061 & 0.825 \\
\hline \multicolumn{5}{|c|}{$K_{\mathrm{s}}$ band $(2.149 \pm 0.309 \mu \mathrm{m})$} \\
\hline \multicolumn{5}{|c|}{ line contribution $22 \%$} \\
\hline H I & 2.166 & -11.549 & 0.0260 & 0.833 \\
\hline He I & 2.058 & -11.769 & 0.0156 & 0.616 \\
\hline
\end{tabular}

Notes. The columns give the element, wavelength, log flux in usual units, the line intensity relative to $\mathrm{H} \beta$, and the combined transmission of the sky, telescope, filter, and detector at this wavelength. Note that the model fluxes have not been multiplied with the transmission. The $Z$, $Y$ and $J$ bands are dominated by one line (but for the $Y$ band it is located at the edge of the filter). $H$ and $K_{\mathrm{S}}$ have a scattering of fainter lines. The total contribution of emission lines to the in-band flux is also given. This is much higher for $Y$ and $Z$ than for the other three bands. Next to the band are indicated the effective wavelengths and the width of VISTA's filters.

- PNe extended (in $\mathrm{H} \alpha$ ) without NIR sources in the regions of their geometrical centre (N/D).

- Objects with unreliable photometry, i.e. large uncertainty in the magnitudes $(>0.2)$, classified as noise or saturated by the pipeline (bad ph.).

Nearly $80 \%$ of the sample is detected in at least one band. Although the integration times in the region of the bulge are shorter than in the plane ( $4 \mathrm{~s}$ and $10 \mathrm{~s}$ per frame for the $K_{\mathrm{s}}$ band), the percentage of objects not detected for the plane and bulge are comparable. We interpret this as that, on average, the bulge images show better contrast than the plane ones.

In general, extended $\mathrm{PNe}$ at $\mathrm{H} \alpha$ are related to unresolved or small extended $\left(\mathrm{PSF}_{\mathrm{PN}} \geq \mathrm{PSF}_{\text {field }}\right)$ sources in the NIR images. In these cases, it is probable that we are measuring the central star of the PN, or emission from its immediate environment. The coordinates are shown in Table 3.

Our catalogue (Table 3) includes $353 \mathrm{PNe}$ with $Z Y J H K_{\mathrm{s}}$ photometrical data. From these, 209 are new NIR detections.
The remaining ones were previously detected and measured by $2 \mathrm{MASS}$, however the VVV photometry improves on these earlier determinations.

\subsection{Comparison between VVV and $2 M A S S$}

To compare the VVV magnitudes with those of 2MASS (The 2MASS Point Source catalogue) ones, we selected objects with $J(2 \mathrm{MASS})<16.0$ and $J(\mathrm{VVV})>11.8, H(2 \mathrm{MASS})<15.5$ and $H(\mathrm{VVV})>11.0, K_{\mathrm{s}}(2 \mathrm{MASS})<14.0$ and $K_{\mathrm{s}}(\mathrm{VVV})>11.0$, according to the upper and lower detection limit, respectively. The brightest objects in VVV were not taken into account since they are near the saturation limit. We also did not consider the weakest PNe in the 2MASS survey because of the uncertainties on the photometry.

The 2MASS Point Source Catalogue was constructed using aperture photometry with 4 " radius (Skrutskie et al. 2006), while we used aperture photometry with a $1.41^{\prime \prime}$ radius. The difference between both magnitudes is about $0.07 \mathrm{mag}$ at $J$ and lower for the other bands (see Table 2 and Fig. 2), therefore we did not apply any additional corrections to the VVV data. This is because the VISTA and 2MASS photometric systems are different, even if the filter names are the same. The VISTA magnitude of an object is not expected to be exactly the same as the 2MASS magnitude (except for an unreddened A0V star) and vice versa.

Examining VVV images showed that the detected NIR emission of the PNe, in general, is point-like or nearly so. The larger aperture of the 2MASS photometry will therefore have little effect on the flux of the object, but it can lead to increased contamination by neighbouring stars, especially in crowded fields (very frequent for the bulge region).

\subsection{The analysis of the VVV photometry}

\subsubsection{The $(J-H)$ vs. $\left(H-K_{s}\right)$ diagram}

All known PNe with photometric data in the VVV are placed in the $J-H$ vs. $H-K_{\mathrm{s}}$ diagram (Fig. 3 ). More than $50 \%$ are objects with original NIR data. The magnitudes of the sources are listed in Table 3. The magnitudes are not dereddened; the reddening vector, obtained from Saito et al. (2012) is indicated by the arrow (its length corresponds to 10 mag extinction in $V$ ).

PNe belonging to the bulge and disc are plotted separately in the upper panel. The lower panel distinguishes point-like from extended objects, as classified by the pipeline. The interpretation of these diagrams was already discussed in Corradi et al. (2008), and our results are consistent (Ramos-Larios \& Phillips 2005; Garcia-Lario et al. 1997; Phillips \& Zepeda-García 2009). The expected colours for unreddened main sequence and giant branch stars are shown in the lower diagram, extending from Vega at $(0,0)$ (in the vicinity of the Rayleigh-Jeans point) to $\left(H-K_{\mathrm{s}}, J-H\right)=(0.5,1.0)$. S-type symbiotic stars (and other emission line stars) are located around $(0.5,1.0)$, and for D-type symbiotics and $\mathrm{T}$ Tau stars a hot dust continuum is added to those to give a broad sequence towards $(2,2)$. Figure 3 shows that the majority of the VVV PNe are located well away from this, and relatively few are found among the stellar locus.

Compared to the sample of Corradi et al. (2008), there is little evidence for significant further reddening. This is not unexpected as the PNe in our sample had previously been discovered in optical surveys.

To investigate if some difference between PNe populations from disc and bulge is present, we distinguished them in the upper panel of Fig. 3. There is an indication for a separation, 
W. A. Weidmann et al.: Planetary nebulae with the VVV Survey
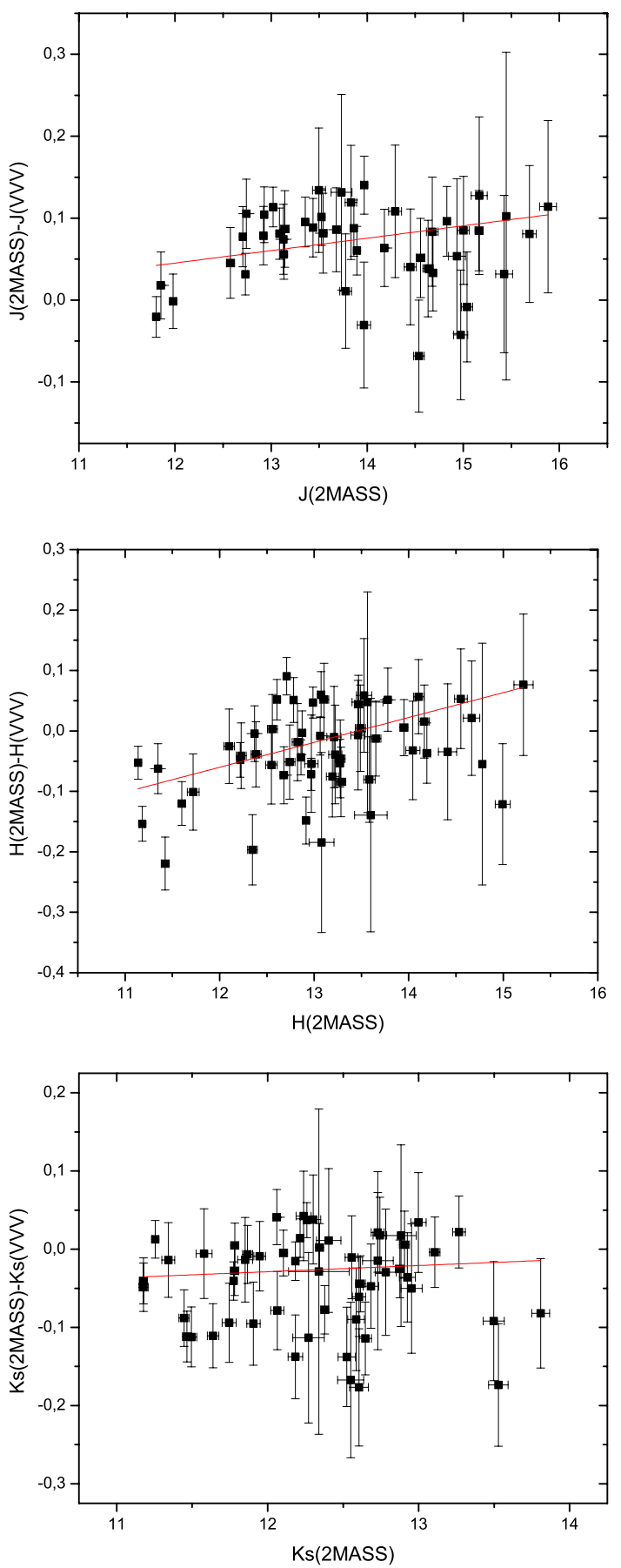

Fig. 2. Comparison between 2MASS and VVV PNe magnitudes.

with the bulge PNe (black dots) slightly bluer both in $J-H$ and $H-K_{\mathrm{s}}$ for objects around the point $\left(H-K_{\mathrm{s}}, J-H\right)=(0.7,0.5)$ where many of the PNe are found. The separation is much less evident among the objects with stellar colours (presumed field stars). This suggests that the separation is not due to systematic differences in extinction.

As mentioned in Sect. 2, the VVV pipeline distinguish between stars (point sources) and "galaxies" (i.e. not point sources). The PNe, according to the flag point-like/not-point-like derived from the VVV pipeline, are plotted in the lower panel of Fig. 3. In general, the analysed sources are interpreted by the pipeline as resolved (66\% of the sample shown in Fig. 3). This could be an interesting aid to find new PNe. The distribution of sources within the $J-H$ vs. $H-K_{\mathrm{s}}$ plane (Fig. 3, lower
Table 2. VVV and 2MASS magnitude comparison (linear fit).

\begin{tabular}{lcccc}
\hline \hline & $N$ & Average & rms & Slope \\
\hline$J(2 \mathrm{MASS})-J(\mathrm{VVV})$ & 46 & +0.066 & 0.049 & $0.015 \pm 0.007$ \\
$H(2 \mathrm{MASS})-H(\mathrm{VVV})$ & 56 & -0.032 & 0.071 & $0.041 \pm 0.010$ \\
$K_{\mathrm{s}}(2 \mathrm{MASS})-\mathrm{K}_{\mathrm{s}}(\mathrm{VVV})$ & 55 & -0.042 & 0.059 & $0.008 \pm 0.010$ \\
\hline
\end{tabular}

panel) shows two concentrations of sources, but the distinction between the stellar and nebular sources only marginally reflects the unresolved/resolved flag. The relation between this flag and the nature of the emission is not obvious.

Interestingly, the stellar locus is sparsely populated compared to other works (for instance Phillips \& Zepeda-García 2009). This fact perhaps shows that VVV, with better resolution, yields improved rejection of unrelated field stars. It is noteworthy that there is a distinct concentration near the locus of unreddened K2 stars. This may indicate these are indeed field stars.

The lower panel of Fig. 3 also shows the loci of freefree/bound-free emission from ionised hydrogen gas. These are calculated for a temperature range from 8400 to $17000 \mathrm{~K}$, at approximately $\left(H-K_{\mathrm{s}}, J-H\right)=(0.7,0.4)$. The actual measurements deviate from this by about $0.5 \mathrm{mag}$, especially in $H-K_{\mathrm{s}}$. This is mainly due to contributions from emission lines. Interestingly, the bulge $\mathrm{PNe}$ tend to be located closer to the bf/ff locus, perhaps reflecting a different emission-line contribution.

We finally compare the observed distribution of PNe with the model of NGC 6720. This gives colours of $\left(H-K_{\mathrm{s}}, J-H\right)=$ $(0.62,0.0)$, towards the bottom range of the observed colours. For a similar model for NGC 7027 (Beintema et al. 1996; Zijlstra et al. 2008) we derive unreddened colours of $\left(H-K_{\mathrm{s}}, J-H\right)=$ $(0.21,0.41)$ also within the general PNe region but close to the blue edge of the PNe distribution. Allowing for a range of extinction values in the observed sample, these two models can cover much of the observed range.

\subsubsection{The $(Y-J)$ vs. $(Z-Y)$ diagram}

Figure 4 shows the distribution of PNe in the newly measured $(Y-J)$ vs. $(Z-Y)$ diagram. We analysed the reddened and dereddened colours (upper and lower panel respectively). In addition, the colours of other emission objects are shown, to be compared with those of PNe. The reddening vector, obtained from Saito et al. (2012), is indicated by an arrow (its length corresponds to 10 mag extinction in $V$ ).

We have extracted from the VVV database the $Z, Y$ and $J$ magnitudes of symbiotic stars (Belczyński et al. 2000), cataclysmic variables (Downes et al. 2001), Be stars (Zhang et al. 2005), and Mira variables (Kharchenko et al. 2002). Although the samples of these objects are not very large, they are useful to estimate the possible overlap with PNe. The distribution of reddened PNe (Fig. 4, upper panel) occupies a wide region in the $(Y-J)$ vs. $(Z-Y)$ diagram, but it is possible to isolate an area to the right of the main sequence (MS) and parallel to the reddening vector (limited by the dashed line), which is not shared by the other objects. Although only $41 \%$ of PNe are placed to the right of the dashed line, the lack of confusion in this region suggests that this diagram, combined with the standard $J H K_{\mathrm{s}}$, will be a useful tool for the identification of new PNe.

The ZYJ diagnostic diagram, using dereddened colours, is shown in Fig. 4 (bottom panel), together with the location of MS stars. The colours are corrected for the interstellar reddening 

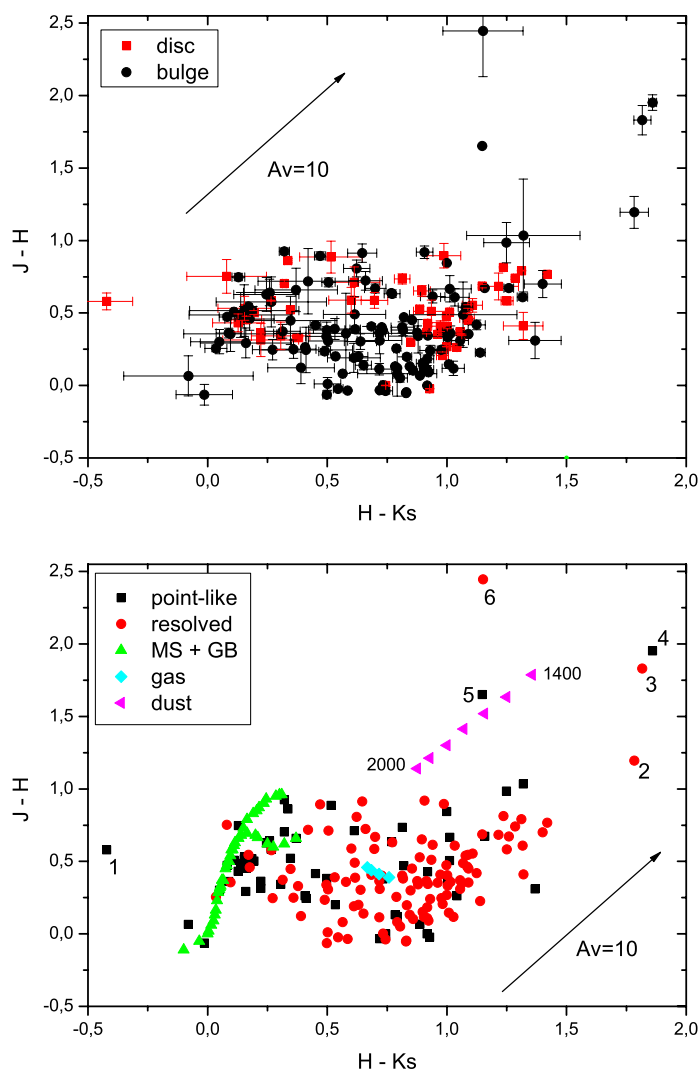

Fig. 3. Upper panel: distribution of PNe according to whether they belong to the plane or bulge. Lower panel: distribution of colour indices $\left(H-K_{\mathrm{s}}\right)$ and $(J-H)$ for the PNe analysed as "galaxy-like" (resolved) by the pipeline, plotted as red circles, point-like sources, plotted as black squares; error bars have been left our for the sake for clarity. The magenta triangles indicate dust emission loci for an emissivity exponent $\gamma=0$ and temperature $T_{\mathrm{d}} \leqslant 2000 \mathrm{~K}$ (in steps of $100 \mathrm{~K}$, Phillips \& Cuesta 1994). Intrinsic stellar indices for MS spectral types (Bessell \& Brett 1988) and the O-type from Martins et al. (2005) are shown by green triangles. Finally, the free-free and free-bound emission from hydrogen ions are shown for the range of 8400 to $17000 \mathrm{~K}$, by the cyan diamond symbols.

using, in general, the extinction coefficient $c$ of Tylenda et al. (1992), and by assuming that $c / E_{B-V}=1.46$ (Pottasch 1984) and taking the standard $A / E_{B-V}=3.1$. The infrared extinction $A_{Z}=0.499 A_{\mathrm{V}}, A_{Y}=0.390 A_{\mathrm{V}}$ and $A_{J}=0.280 A_{\mathrm{V}}$ were taken from Saito et al. (2012). We estimate an uncertainty of $10 \%$ on the extinction coefficients $c$ (see Fig. 4).

There is some overlap in colours between MS stars and unreddened PNe, and this overlap is increased by reddening (top panel). This diagram does not allow us to differentiate PN from MS stars with high efficiency. However, it is evident that the PNe tend to be grouped in a bounded region, and there is a welldefined region containing about half the PNe which is not affected by confusion with the depicted types of objects.

The $Y$ and $Z$ bands have strong contributions from emission lines and have less continuum as the bands are relatively narrow (as compared to $J H K_{\mathrm{s}}$ ). The $J$-band contains a strong $\mathrm{Pa} \beta$ line but also more continuum, as the band is wider. The $Z-Y$ colour can become negative because the $Z$-band contains a strong [SIII] line.
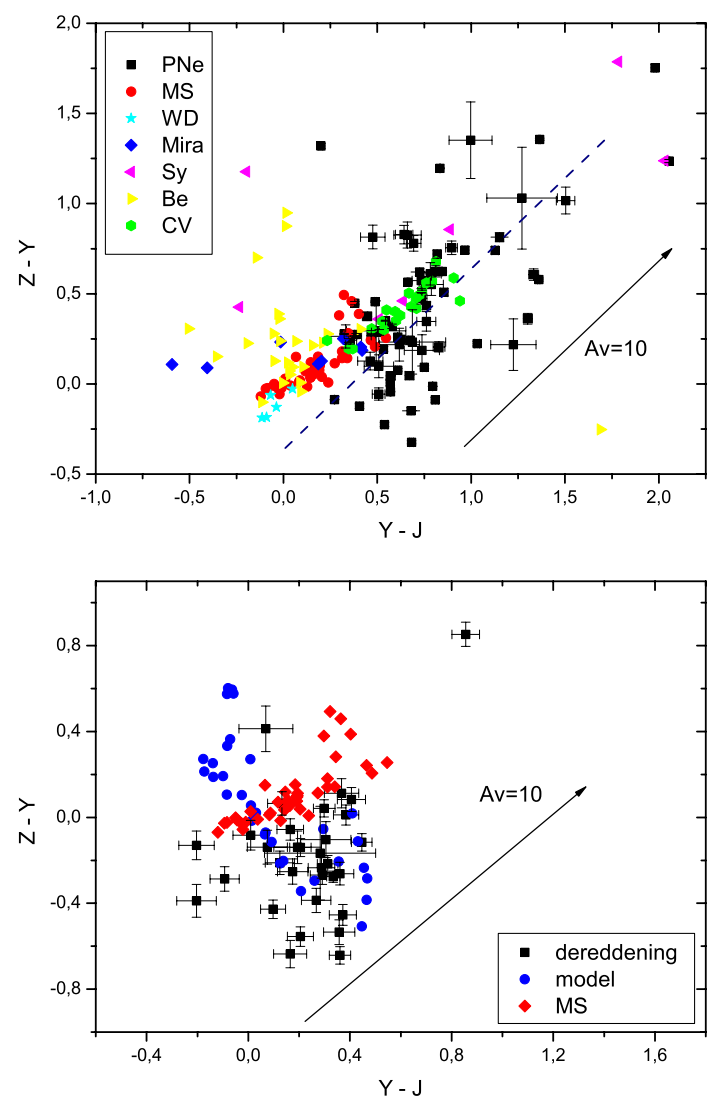

Fig. 4. Distribution of the colour indices $(Y-J)$ and $(Z-Y)$. The upper panel show all detected $\mathrm{PNe}$ without applying any extinction correction. Other symbols show other emission-line stars, main sequence stars (Hewett et al. 2006) and white dwarfs (taken from the standard database of MKO) as indicated. The bottom panel shows dereddened colours for the PNe, using the reddening measured from the hydrogen lines. The distribution of the colour indices $(Y-J)$ and $(Z-Y)$ for a model grid of planetary nebulae is shown in blue.

\subsubsection{Notes on individual objects}

A number of objects show unexpected colours. These are labelled by number in Fig. 3, lower panel. The label number is indicated below, after the name of the PN (see Fig. 5).

PN G347.4+01.8 - (1) This shows a very blue $\left(H-K_{\mathrm{S}}\right)$ colour. The $\mathrm{H} \alpha$ image shows a bipolar morphology (Parker et al. 2006), we identify a VVV NIR source at the geometric centre of the belt, however this could be a spurious star.

PN G001.9+02.3.- (2) The colour is indicative of a highly reddened PN. However, we could not reject a very young PN (Ramos-Larios et al. 2009; Lewis 2006).

PN G355.2-02.0.- (3) Similar to the previous object, the colour indicates high reddening. The object has a high excitation class (Parker et al. 2006). However, in agreement with Viironen et al. (2009b, Fig. 1) this could be a D-type symbiotic star.

PN G355.0-03.3.- (4) Suspected to be a symbiotic star (Miszalski et al. 2009). 

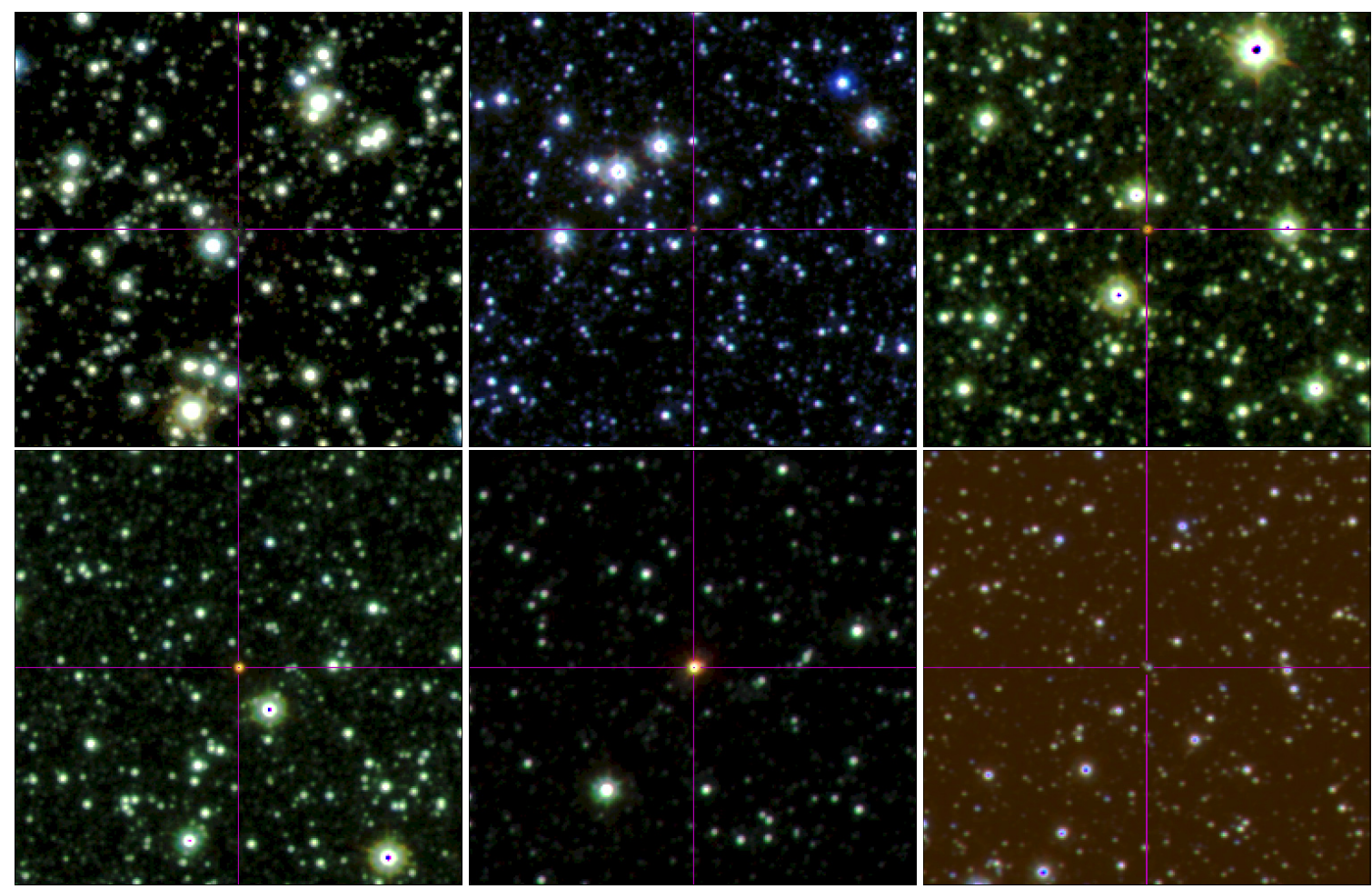

Fig. 5. $J H K_{\mathrm{s}}$ colour composite images showing the six stars (red cross) described at Sect. 4.3.3, range from top left to bottom right. The size of images are 1.4'.

PN G356.9-05.8.- (5) Its colours can be explained as dominated by hot dust. It is suspected to be a symbiotic star (Miszalski et al. 2009).

PN G000.1-01.0.- (6) This object has unusual colours. It is a poorly studied object, and perhaps it is not a PN.

\subsection{Model distribution}

We can again compare the colours of the NGC 6720 model with the observed distribution. The synthetic colour of $(Y-J, Z-Y)=$ $(0.12,0.21)$. The NGC 7027 model has very similar (unreddened) colours of $(Y-J, Z-Y)=(0.10,0.24)$. These fall at the edge of the observed distribution (upper panel of Fig. 4). NGC 7027 is a young, high density PN, whilst NGC 6720 is evolved and has low density. It is therefore not immediately obvious what causes any differences with the observed distribution.

To explore this further, we computed a Cloudy model grid, and for each model in the grid calculated the $Y-J$ and $Z-Y$ colours. The grid is defined by varying the effective temperature of the star and the hydrogen density of the nebula. The grid runs from $\log T_{\text {eff }}=4.7$ to 5.4 in steps of $0.1 \mathrm{dex}$ (temperatures from $50 \mathrm{kK}$ to $250 \mathrm{kK}$ ), and from $\log n_{\mathrm{H}}=2$ to 5 in steps of $1 \mathrm{dex}$. The model nebula has exactly $N_{\mathrm{H}}=10^{57}$ atoms corresponding to a mass of approximately $1.2 M_{\odot}$, and all models are calculated up to the outer radius with inclusion of molecular hydrogen in a PDR (Photon Dominated Regions) region, if present.

The lower panel of Fig. 4 shows the distribution of points in the $(Y-J)$ and $(Z-Y)$ plane. The models follow the lower edge of the observed $\mathrm{PNe}$ distribution in the upper panel of Fig. 4. Together with a range of extinction in the VVV sample, the observed distribution can be well explained. There is some discrepancy in the lower panel, in that some dereddened data fall below the model grid. This may reflect limitations in the model grid, such as a constant nebular mass and constant abundances (especially for sulphur). It is also possible that in some cases the extinction coefficient $c$ of Tylenda et al. (1992) is over-estimated, or that the scaling from $\mathrm{H} \alpha / \mathrm{H} \beta$ to optical and near-infrared broad-band colours is also affected by the shape of the reddening law. Ruffle et al. (2004) have shown indications that the common assumption of $R=3.1$ used here may not always be appropriate for known PNe.

The model grid extends to a region above the stellar locus in the $(Y-J, Z-Y)$ diagram. There are almost no observed $\mathrm{PNe}$ in this region where many of the models fall. These models are those with hottest ionizing stars. At all densities, the $Z-Y$ colour increases with increasing stellar temperature, as shown in Fig. 6. The largest change occurs between temperatures of $10^{5}$ and $1.25 \times 10^{5} \mathrm{~K}$. The $Y-J$ curve is largely the mirror image of the $Z-Y$ curve. This indicates that the change is caused by a high excitation line in the $Y$ band. The He I $1.083 \mu \mathrm{m}$ lines decreases between $T_{\text {eff }}=10^{5}$ en $10^{5.2} \mathrm{~K}$, and the He II $1.012 \mu \mathrm{m}$ becomes much stronger. Both lines are in the $Y$ band, but the latter dominates because of the much higher transmission efficiency at its wavelength (Table 1). The effect is strongest for the lower density models as these are density bounded, and lack any lower ionisation regions. Note that the model in Table 1 is ionisation bounded.

The four model points at $(Y-J, Z-Y)=(-0.08,0.55)$ are low density models with $n_{\mathrm{H}}=10^{2} \mathrm{~cm}^{-3}$. These are fully ionised and have very large predicted diameters ( 22 arcsec) at the distance of the bulge. The three models just below this have $n_{\mathrm{H}}=10^{3} \mathrm{~cm}^{-3}$, are also fully ionised with predicted diameters of 10 arcsec. The nebulae which are missing from the observed distribution are those with very hot stars and large to very large nebulae. Such nebulae may have been removed from the sample (as described above), may not have been incuded in the VVV catalogues, or 

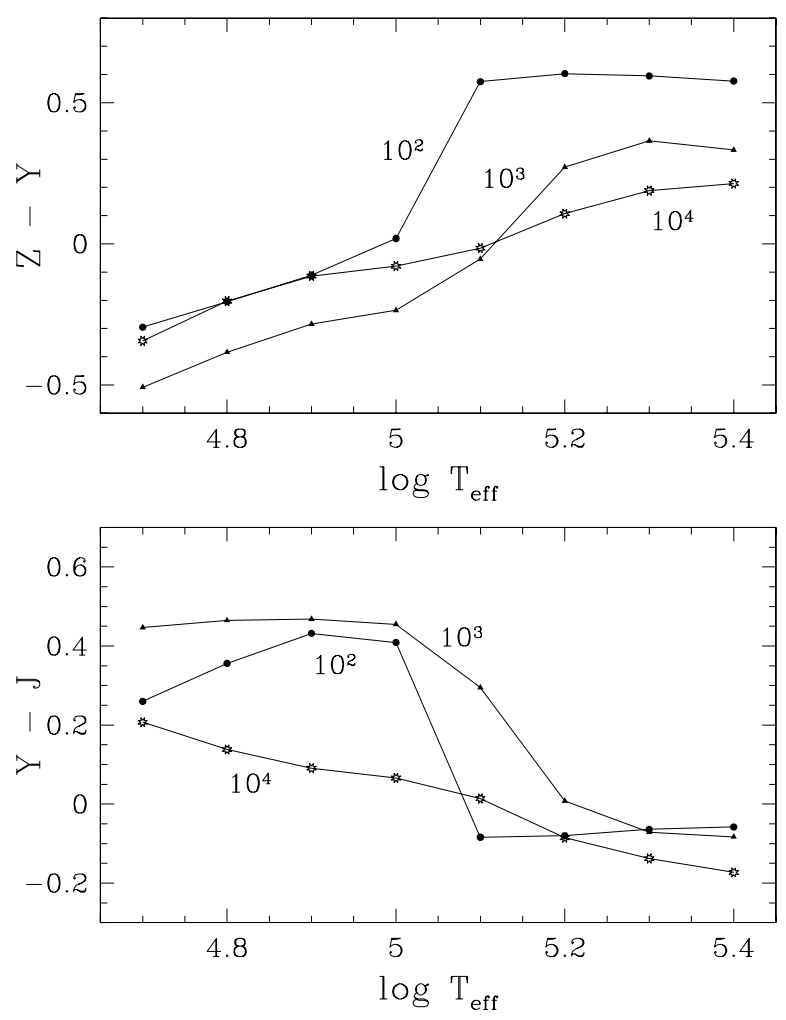

Fig. 6. Distribution of the colour indices $(Y-J)$ and $(Z-Y)$ as function of stellar temperature, for the Cloudy model grid described in the paper. Each curve is for a fixed density, indicated by the adjacent label.

their colours have been affected by the use of an aperture much smaller than the size of the nebula.

\section{Conclusions}

The VVV survey is shown to be sensitive to planetary nebulae. Of the 579 known PNe (excluding 75 highly extended objects) in the covered region, 353 have been detected in at least one filter. The majority of these (209) are new near-infrared detections. The NIR images, to the naked eye, suggest compact, point-like sources; however the VVV pipeline classifies a high percentage of PNe as extended sources. Aperture photometry was obtained from the pipeline catalogues. In the $J, H, K_{\mathrm{s}}$ bands, the photometry agrees well with $2 \mathrm{MASS}$ data where available, in most cases within $0.1 \mathrm{mag}$, even though 2MASS and VISTA are each on their own photometric system.

We use a photo-ionisation model of NGC 6720 (the Ring nebula) to calculate the flux contributions from emission lines and from the continuum, for each of the five filters. The line contribution decreases with increasing wavelength, from $55 \%$ in $Z$ and $Y$, to $22 \%$ in $K_{\mathrm{s}}$. The precise contribution depends on the excitation of the nebula, especially at $Z$ and $Y$ where the dominant lines are from sulphur and helium, respectively. The $J, H$, $K_{\mathrm{s}}$ bands are dominated by hydrogen, where it should be noted that molecular hydrogen can also contribute at $K_{\mathrm{s}}$. Electron temperature and elemental abundances may also affect the relative contributions.

We explore two diagnostic diagrams: $H-K_{\mathrm{s}}$ vs. $J-H$, and $Y-J$ vs. $Z-Y$. In the former, the PNe are well separated from the stellar locus and from emission line stars. The PNe are located around the colours of the continuum from ionised hydrogen plasmas. Bulge PNe tend to be located closer to these colours than do PNe of the Galactic disc. The reason for this is not clear, but metallicity may play a role. The $Y-J$ vs. $Z-Y$ diagram shows a broad spread in colours, and PNe have more overlap with the stellar locus. About half fall in a region of the diagram where there is little confusion, so that this diagram can still be a useful tool in finding and classifying PNe.

A model grid was calculated using the Cloudy photoionisation code, covering a wide range of stellar temperatures and electron densities, and assuming standard PN abudances. The model spectra were convolved with the VISTA response functions to predict colours. The models show a range of $Y-J$ $Z-Y$ colours whith a sequence aproximately perpendicular to the stellar locus. The sequence follows the lower boundary of the distribution of observed PNe; the observed distribution can be reproduced by reddening the model nebulae. There are no observed PNe in the region above the stellar locus, where a significant fraction of the model nebulae are located. These are related to nebulae with hot central stars $\left(T>10^{5} \mathrm{~K}\right)$, where the He II line at $1.012 \mu \mathrm{m}$ becomes the dominant line in $Y$-band. The missing objects correspond to very large, fully ionised nebula with hot stars which are absent from our selected sampe.

The reddening coefficients for many of the observed PNe have been published by Tylenda et al. (1992). When we use these to de-redden our sample, the resulting distribution shows that some objects fit the models very well, but a fraction have colours which are bluer than shown by any of the models. The discrepancy is up to $0.4 \mathrm{mag}$ in $Y-Z$ and $J-Y$. This suggests that the extinction in some nebulae may have been over-estimated. Alternatively, as suggested by Ruffle et al. (2004), uncertainties in the $R_{V}$-value (assumed to be 3.1) may have an effect.

The VVV survey is shown to be a powerful tool to study planetary nebulae in the crowded and extincted region of the galactic plane. The large majority of known objects ware detected, but in these regions, many $\mathrm{PNe}$ are expected to have remained undiscovered. The results presented here will help locating this missing population in the VVV database.

Acknowledgements. We thank the anonymous referee whose very useful remarks helped us to substantially improve this paper. We acknowledge Jim Emerson for comments that helped to improve the paper. This research has made use of the Aladin and SIMBAD database, operated at CDS, Strasbourg, France. This publication makes use of data products from the Two Micron All Sky Survey, a joint Project of the University of Massachusetts and IPAC/CALTECH, funded by NASA and NSF. We gratefully acknowledge use of data from the ESO Public Survey programme ID 179.B-2002 taken with the VISTA telescope, data products from the Cambridge Astronomical Survey Unit (CASU), and from the VISTA Science Archive at the Wide Field Astronomy Unit (WFAU). P.v.H. acknowledges support from the Belgian Science Policy Office through the ESA PRODEX program. D.M. gratefully acknowledges funding from the FONDAP Center for Astrophysics 15010003, the BASAL CATA Center for Astrophysics and Associated Technologies PFB-06, the MILENIO Milky Way Millennium Nucleus from the Ministry of Economy's ICM grant P07-021-F, and Proyecto FONDECYT Regular No. 1090213 from CONICYT and the astro-Enginiering center at the Universidad Católica (AIUC).

\section{Appendix A: Discussion about aperture}

As we are dealing with extended sources, some special care had to be taken. Thus, we characterised the object sizes by means the Petrosian radius (Petrosian 1976; Yasuda et al. 2001) provided by CASU (see Table 4). However, the relation between a measured size and the real size of an extended nebula depends on the resolution/seeing versus actual size, and on the assumed geometry. There is a discussion on this in van Hoof (2000). 
W. A. Weidmann et al.: Planetary nebulae with the VVV Survey
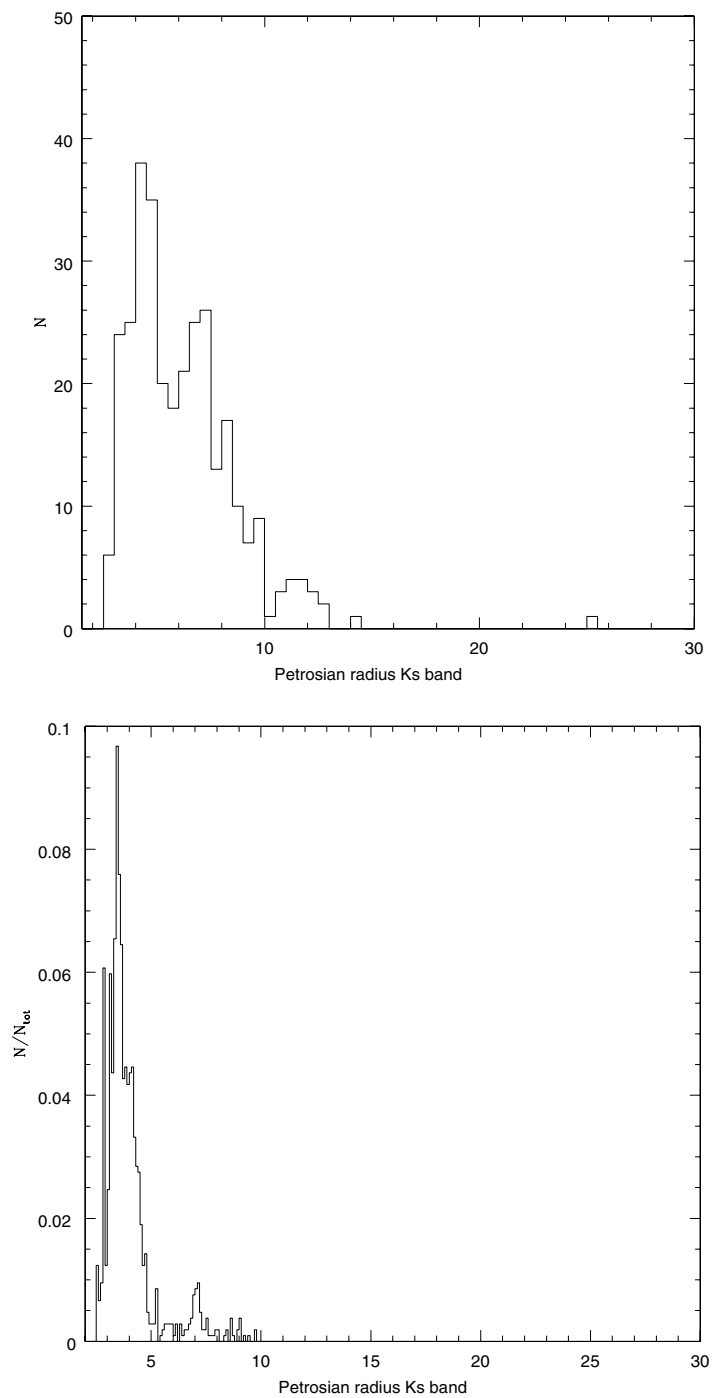

Fig. A.1. Distribution of Petrosian radii, in pixels. Upper panel, distribution for our sample of PNe (data from Table 4). Lower panel, distribution for a sample of sources clasiffied like stars by CASU.

According to Fig. A.1 (upper panel), roughly $80 \%$ of PNe in our sample have a Petrosian radius less than $8 \mathrm{px}$, equivalent to $2.6^{\prime \prime}$. In this sense, the magnitudes for our sample of $\mathrm{PNe}$ should be those of aperture-6 $\left(=2.83^{\prime \prime}\right)$ in the CASU catalogue.

In order to evaluate how important the differences between magnitudes obtained with an aperture 4 and 6 are, we assemble a histogram of aperMag4 - aperMag6 (Fig. A.2). Clearly, differences between both magnitudes are important. The tail towards brighter aperture- 6 magnitudes may indicate the effect of extended sources and the presence of neighbour stars within the aperture. To evaluate this later hypothesis, we computed the number of stars that are closer than 2.6" to each PNe. Surprisingly, about $70 \%$ of the sample do not have any contaminant star (Fig. A.3), pointing out that the differences should be due to other effects.

Alternatively, we analysed the sky subtraction performed by the CASU pipeline and found that the error introduced by this task is very significant. The weakest sources, as the PNe in our sample, are more affected by sky subtraction (see Fig. A.2). Also, we note that the magnitudes obtained with aperture- 4 compare better with those of 2MASS (Fig. 2) than the aperture-6

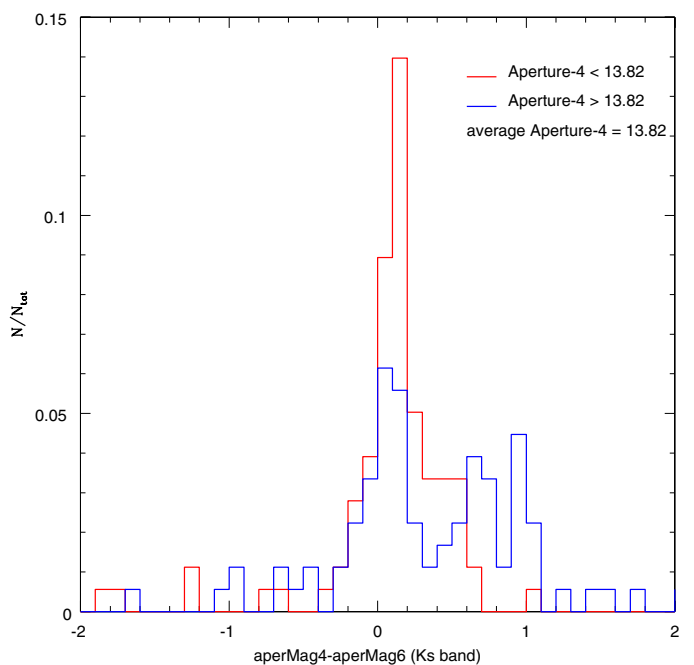

Fig. A.2. Comparison between magnitudes with aperture-6 (2.83") and aperture-4 (1.41") for our sample of PNe.

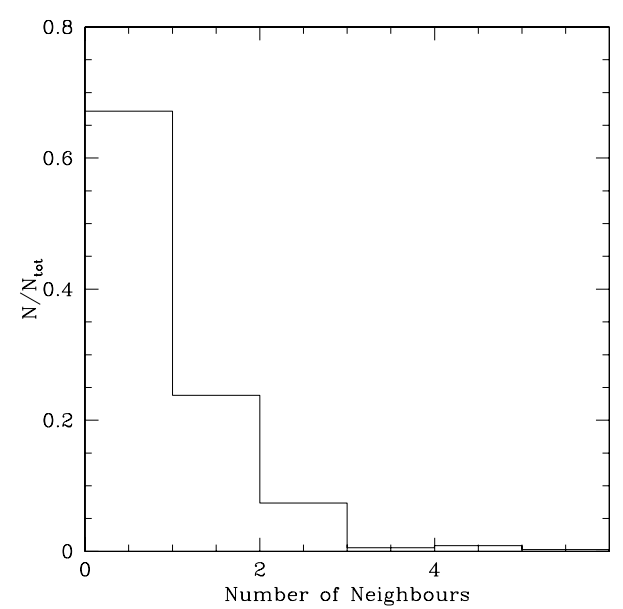

Fig. A.3. Distribution of number of contaminant stars around each $\mathrm{PNe}$ of our sample.

ones, and the distribution of PNe in the $J-H$ vs. $H-K_{\mathrm{s}}$ diagram is more scattered.

Although it seems more appropriate to obtain the magnitudes with an aperture of $2.83^{\prime \prime}$, increasing aperture implies increasing uncertainty in the magnitudes (due the sky subtraction), and the magnitudes obtained with larger aperture are affected by larger errors. In this sense, we demonstrate that the more accurate magnitudes are those computed with the CASU pipeline using the aperture of $1.41^{\prime \prime}$.

\section{References}

Acker, A., Marcout, J., Ochsenbein, F., et al. 1992, The Strasbourg-ESO Catalogue of Galactic Planetary Nebulae. Parts I, II (ESO, Garching), eds. A. Acker, J. Marcout, F. Ochsenbein, et al.

Allen, D. A. 1973, MNRAS, 161, 145

Beaulieu, S. F., Dopita, M. A., \& Freeman, K. C. 1999, ApJ, 515, 610

Beintema, D. A., van Hoof, P. A. M., Lahuis, F., et al. 1996, A\&A, 315, L253

Belczyński, K., Mikołajewska, J., Munari, U., Ivison, R. J., \& Friedjung, M. 2000, A\&AS, 146, 407

Bessell, M. S., \& Brett, J. M. 1988, PASP, 100, 1134

Corradi, R. L. M., Rodríguez-Flores, E. R., Mampaso, A., et al. 2008, A\&A, 480, 409

Cross, N. J. G., Collins, R. S., Mann, R. G., et al. 2012, A\&A, 548, A119

Dalton, G. B., Caldwell, M., Ward, A. K., et al. 2006, in SPIE Conf. Ser., 6269

Downes, R. A., Webbink, R. F., Shara, M. M., et al. 2001, PASP, 113, 764

Drew, J. E., Greimel, R., Irwin, M. J., et al. 2005, MNRAS, 362, 753 
Emerson, J., \& Sutherland, W. 2010, The Messenger, 139, 2

Emerson, J., McPherson, A., \& Sutherland, W. 2006, The Messenger, 126, 41

Epchtein, N., de Batz, B., Capoani, L., et al. 1997, The Messenger, 87, 27

Ferland, G. J., Korista, K. T., Verner, D. A., et al. 1998, PASP, 110, 761

Garcia-Lario, P., Manchado, A., Pych, W., \& Pottasch, S. R. 1997, A\&AS, 126, 479

Gonzalez, O. A., Rejkuba, M., Zoccali, M., Valenti, E., \& Minniti, D. 2011, A\&A, 534, A3

Hewett, P. C., Warren, S. J., Leggett, S. K., \& Hodgkin, S. T. 2006, MNRAS, 367,454

Hyung, S., Aller, L. H., Feibelman, W. A., \& Lee, S.-J. 2001, ApJ, 563, 889

Irwin, M. J., Lewis, J., Hodgkin, S., et al. 2004, in SPIE Conf. Ser. 5493, eds. P. J. Quinn, \& A. Bridger, 411

Jacoby, G. H., \& Van de Steene, G. 2004, A\&A, 419, 563

Kerber, F., Mignani, R. P., Guglielmetti, F., \& Wicenec, A. 2003, A\&A, 408, 1029

Kharchenko, N., Kilpio, E., Malkov, O., \& Schilbach, E. 2002, A\&A, 384, 925

Kinman, T. D., Feast, M. W., \& Lasker, B. M. 1988, AJ, 95, 804

Lewis, B. M. 2006, in Planetary Nebulae in our Galaxy and Beyond, eds. M. J. Barlow, \& R. H. Méndez, IAU Symp., 234, 449

Maciel, W. J., \& Costa, R. D. D. 2003, in Planetary Nebulae: Their Evolution and Role in the Universe, eds. S. Kwok, M. Dopita, \& R. Sutherland, IAU Symp., 209, 551

Martins, F., Schaerer, D., \& Hillier, D. J. 2005, A\&A, 436, 1049

Minniti, D., Lucas, P. W., Emerson, J. P., et al. 2010, New Astron., 15, 433

Miszalski, B., Parker, Q. A., Acker, A., et al. 2008, MNRAS, 384, 525

Miszalski, B., Acker, A., Moffat, A. F. J., Parker, Q. A., \& Udalski, A. 2009, A\&A, 496, 813
Miszalski, B., Napiwotzki, R., Cioni, M.-R. L., et al. 2011, A\&A, 531, A157 Parker, Q. A., Phillipps, S., Pierce, M. J., et al. 2005, MNRAS, 362, 689 Parker, Q. A., Acker, A., Frew, D. J., et al. 2006, MNRAS, 373, 79

Pena, M., \& Torres-Peimbert, S. 1987, Rev. Mex. Astron. Astrofis., 14, 534 Petrosian, V. 1976, ApJ, 209, L1

Phillips, J. P., \& Cuesta, L. 1994, A\&AS, 104, 169

Phillips, J. P., \& Zepeda-García, D. 2009, MNRAS, 394, 1875

Pottasch, S. R., ed. 1984, Planetary nebulae - A study of late stages of stellar evolution, Astrophys. Space Science Lib., 107

Ramos-Larios, G., \& Phillips, J. P. 2005, MNRAS, 357, 732

Ramos-Larios, G., Guerrero, M. A., Suárez, O., Miranda, L. F., \& Gómez, J. F. 2009, A\&A, 501, 1207

Rauch, T. 2003, A\&A, 403, 709

Rudy, R. J., Lynch, D. K., Mazuk, S., Puetter, R. C., \& Dearborn, D. S. P. 2001 , AJ, 121,362

Ruffle, P. M. E., Zijlstra, A. A., Walsh, J. R., et al. 2004, MNRAS, 353, 796

Saito, R. K., Hempel, M., Minniti, D. et al. 2012, A\&A, 537, A107

Skrutskie, M. F., Cutri, R. M., Stiening, R., et al. 2006, AJ, 131, 1163

Tylenda, R., Acker, A., Stenholm, B., \& Koeppen, J. 1992, A\&AS, 95, 337

van Hoof, P. A. M. 2000, MNRAS, 314, 99

van Hoof, P. A. M., van de Steene, G. C., Barlow, M. J., et al. 2010, A\&A, 518, L137

Viironen, K., Greimel, R., Corradi, R. L. M., et al. 2009a, A\&A, 504, 291

Viironen, K., Mampaso, A., Corradi, R. L. M., et al. 2009b, A\&A, 502, 113

Whitelock, P. A. 1985, MNRAS, 213, 59

Yasuda, N., Fukugita, M., Narayanan, V. K., et al. 2001, AJ, 122, 1104

Zhang, P., Chen, P. S., \& Yang, H. T. 2005, New Astron., 10, 325

Zijlstra, A. A., van Hoof, P. A. M., \& Perley, R. A. 2008, ApJ, 681, 1296 